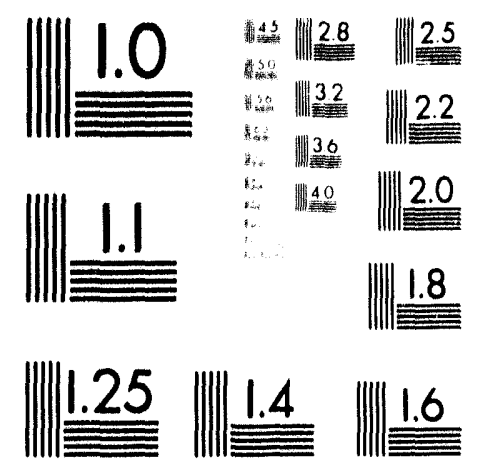



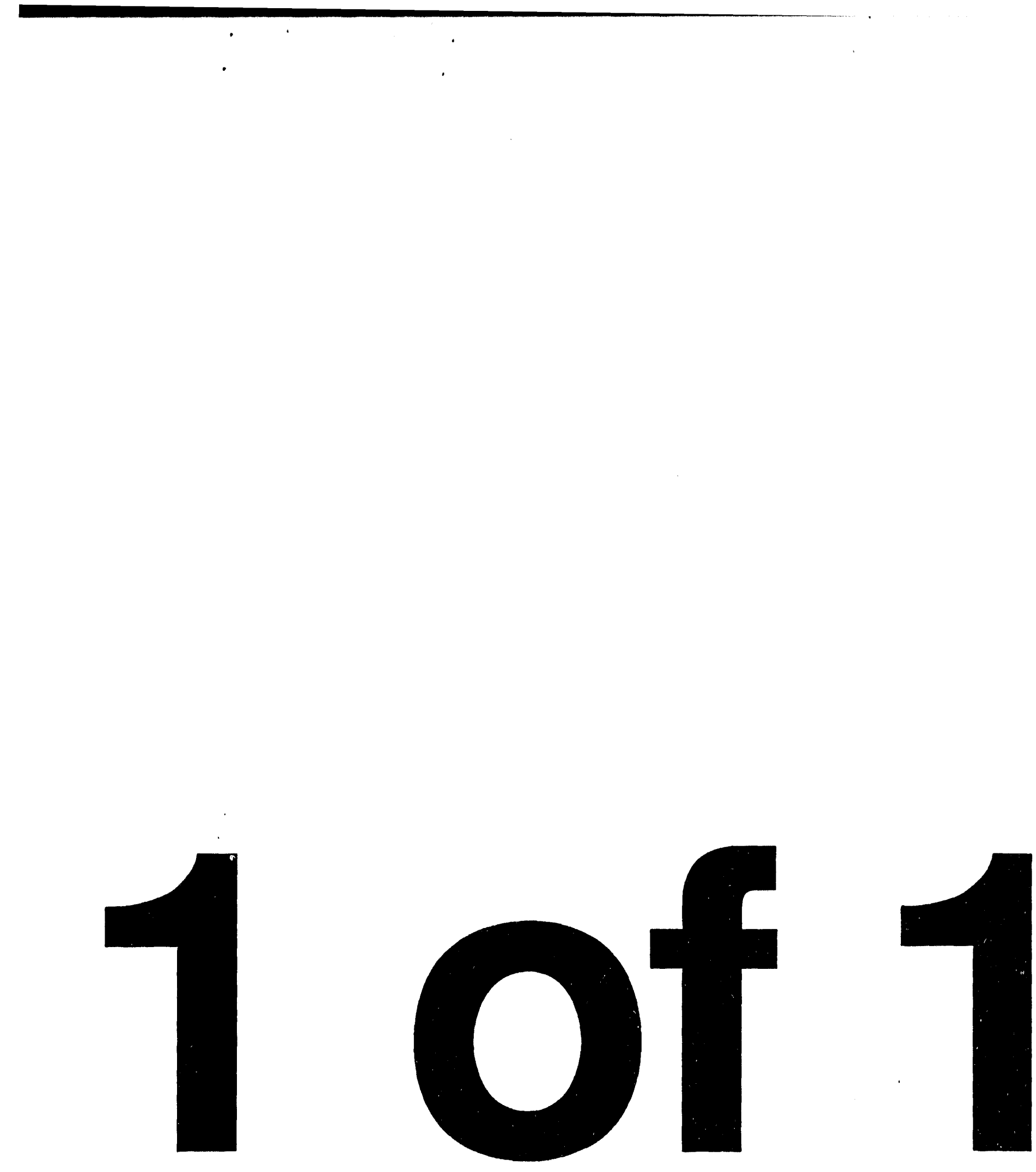


\title{
Beam-Quality Measurements for Materials Processing Lasers and the Proposed ISO Standard
}

\author{
Phillip W. Fuerschbach \\ Marce'ino Essien \\ Sandia Nati il Laboratories \\ Albuquerque, New Mexico 87185-5800
}

\begin{abstract}
Laser beam-quality can indicate the maximum power density that can be obtained with a specific laser and serves as a figure-of-merit when comparing lasers, calibrating lasers, and in assessing a laser's operating condition. Measurements of laser beam-quality for a 1000 watt continuous wave $\mathrm{CO} 2$ laser and a 400 watt pulsed $\mathrm{Nd}$ :YAG laser have been completed. These measurements were made with two different type instruments: 1) a specially constructed apparatus that uses an integrating sphere and diamond apertures and (2) a commercially available instrument that incorporates a scanning and spinning hollow needle. Laser beam-quality and the propagation constants have been determined using a non-linear curve-fitting technique and the new proposed ISO standard for measuring laser beam-quality. The curve fitting analysis and the ISO analysis were found to produce approximately the same values for beam-quality and the propagation constants. However, a comparison of the experimental procedures required with the two techniques showed significant differences. The utility of the ISO technique was limited by it's experimental procedural restrictions. The importance of spherical aberration in distorting laser beam-quality measurements and in limiting the application of beamquality to laser spotsize prediction is also detailed.
\end{abstract}

\section{Introduction}

Laser beam materials processing is primarily selected for manufacturing because it can produce very high power densities that can melt, vaporize, and ablate many different materials. In welding, the high power density of lasers can produce deep penetration welds which have smaller fusion zones than can welding processes which have lower power density. Optimum laser welding occurs within a specific range of laser power densities. Too low a power density results in significant reflectivity losses (Ref. 1). Too high a power density leads to excessive metal spatter or plasma shielding of the beam (Ref. 2). By varying the focusing lens focal length, laser welders can select the power density required for a particular material and joint design, since focus spotsize is directly proportional to focal length. Measurements of the focused laser spotsize are therefore critical in laser welding for calibration in a manufacturing application and are a "best practice" for process control.

The propagation of a high power laser beam through a focusing lens is illustrated in Figure 1 and is described by the accompanying equation (Ref. 3 ), where $w_{0}$ is the focused spotsize at the focal plane, $z_{0}$ is the position of the minimum spotsize relative to the lens, and $\mathrm{M}^{2}$ is a propagation factor that represents the focusability of the laser beam relative to a beam limited solely by diffraction. Since $M^{2}$ represents the potential of a laser beam, it is often referred to as the beam-quality although the product of $w_{0}$ and $\theta_{0}$ (the cone
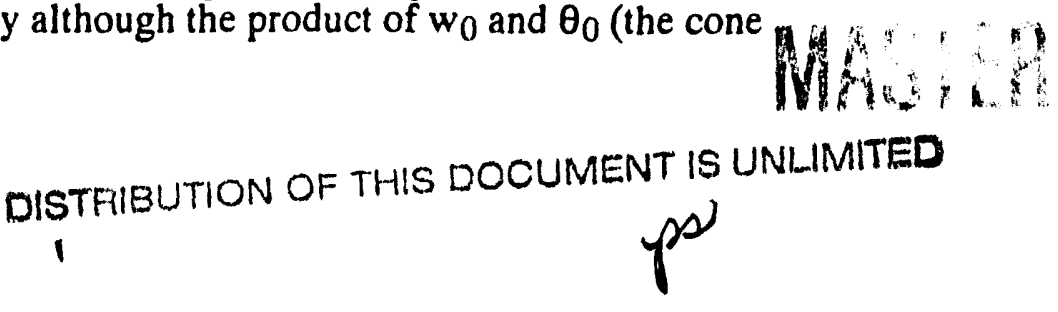
angle of the beam focused by the lens) also provides a measure of beam-quality. One can see from the figure that by determining the functional relationship between focused spotsize and distance to the lens, these propagation constants are also determined. An important corollary to this fact is that by knowing these constants the propagation of the laser beam through aberration free focusing optics can be accurately predicted.

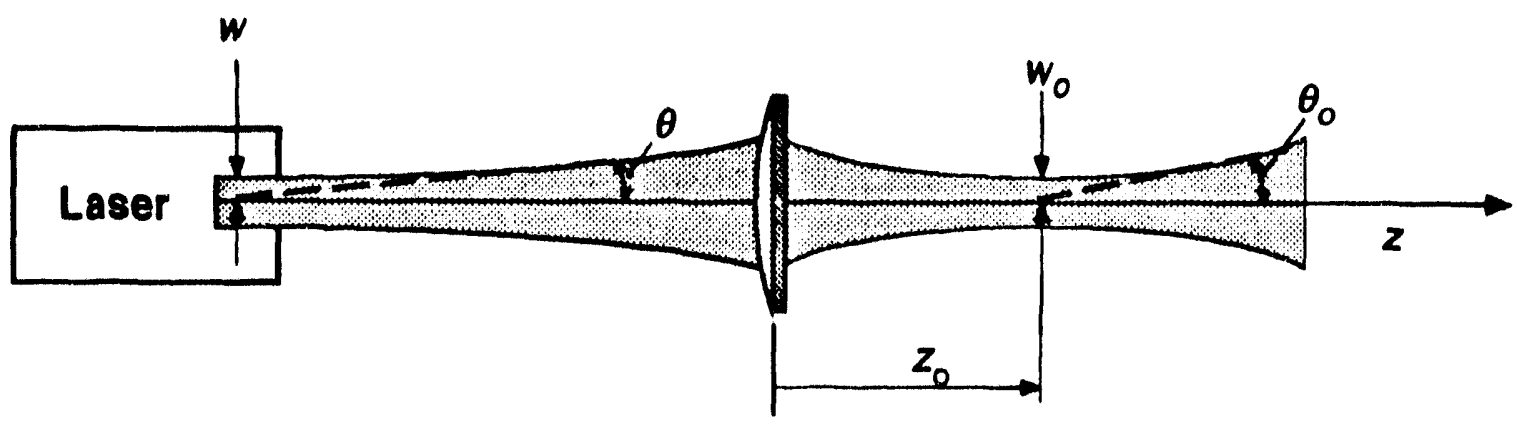

[1]

$$
w^{2}(z)=w_{o}^{2}+M^{4}\left(\frac{\lambda^{2}}{\pi^{2} w_{o}^{2}}\right)\left(z-z_{o}\right)^{2}
$$

[2]

$$
w_{o} \theta_{o}=w \theta
$$

\section{Fig. 1-The equations for propagation of a multi-mode laser beam through a focusing lens.}

Laser beam-quality can indicate the maximum power density that can be obtained with a specific laser and serves, as a figure-of-merit when comparing lasers, calibrating lasers, and in assessing a lasers operating condition. But the significant experimental difficulties inherent in measurements of focused laser spotsize have kept many users of materials processing lasers unaware of their laser's intrinsic beam-quality. In recent years several manufacturers have produced instruments that can make these measurements more practical. A new proposed ISO standard (Ref. 4) for measuring laser beam-quality details a method for performing measurements of the focused laser spotsize and thereby determine the beam-quality. The standard is intended to help users in making these measurements and to assure uniformity in reported values.

Sandia National Laboratories over the years has tested several spotsize measurement techniques (Ref. 5,6) in support of ongoing laser welding research experiments. Recently, an extensive series of spotsize measurements has been completed for a 1000 watt continuous wave $\mathrm{CO}_{2}$ laser. These measurements were made with two different type instruments: 1 ) a specially constructed instrument that uses an integrating sphere and diamond apertures; and (2) a commercially available instrument that incorporates a scanning and spinning hollow needle. A comparison of the two measurement instruments and the results will be presented in this report. A personal computer (PC) based technique for analyzing focused laser spotsize measurements and determining beam-quality was used in the data analysis. Since the ISO standard became available at the time of the measurements, it was decided to try to make some of these measurements using the newly proposed ISO procedures on the same materials 
processing laser. A comparison of the two analysis techniques and their results will also be presented in this report.

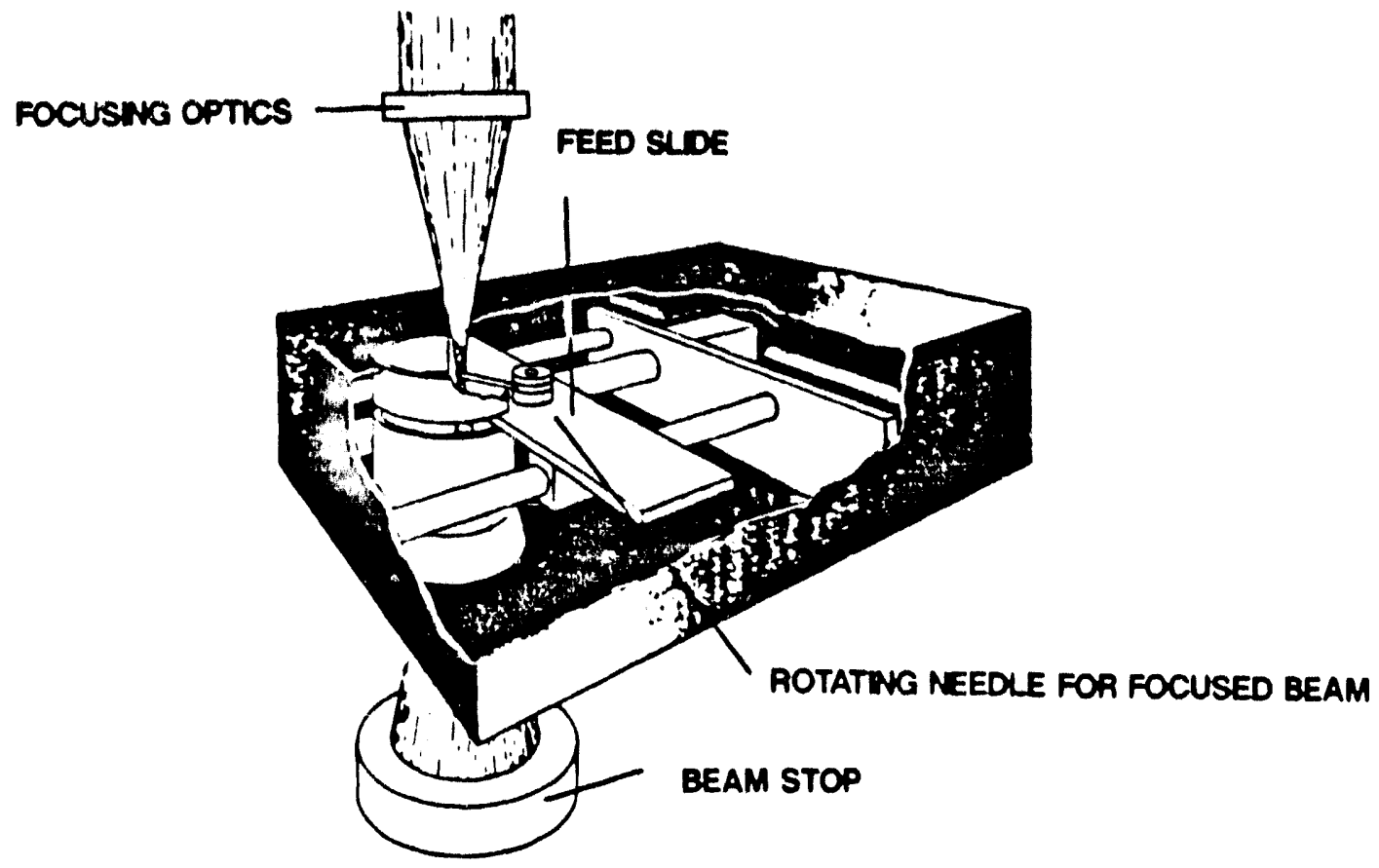

Fig. 2-Schematic diagram of Prometec Laserscope spotsize measuring instrument.

\section{Experimental}

A Prometec Laserscope ${ }^{1}$ was used with a Photon Sources V1200 slow flow continuous wave $\mathrm{CO}_{2}$ laser for the majority of the beam-quality measurements made in this study. The operation of the instrument is illustrated in Figure 2. As shown in the figure the instrument is capable of measuring either a focused or unfocused beam. In either case, a rotating hollow needle, with a pinhole facing up in the end, is rastered under the laser beam in order to map the spatial intensity distribution. The light energy that is captured by the pinhole aperture is reflected down the shaft of the hollow needle where it is measured by a pyroelectric detector. The resulting energy versus position information is stored in the memory of an IBM PC computer. The resulting three dimensional representation of the beam intensity distribution is used to determine the region in which $86 \%$ of the total beam power is contained. The spotsize measured is the radius of a circle having the same area as this region. Because of the way the beam is scanned, the instrument at present is not practicable for measurements of pulsed lasers.

To determine beam-quality in the traditional manner (Ref. 7 ) and with the proposed ISO standard, separate measurements are required for the minimum spotsize, its position relative to the lens, and a second spotsize measurement a known distance away from the minimum spotsize. Measurements of the beam propagation constants were made with the ISO technique as detailed by Wright.(Ref. 4) During the course of the experiments, it was found that the measurements of the beam far from the focal plane did not correlate well with the measurements taken near the focal plane. This caused an experimental

1 Reference 10 a commercial product implies no endorsement by Sandia National Laboratories or the Department of Energy. 
difficulty with the ISO procedure because of the ISO requirement that the measurement made away from the focal plane be taken at the position where the beam radius is between 1.5 and 1.6 times the focal plane value. Measurements taken at this position did not correlate well with the measurements taken at the focal plane. As a result, only the short focal length lenses tested with the Laserscope could be analyzed with the ISO technique.

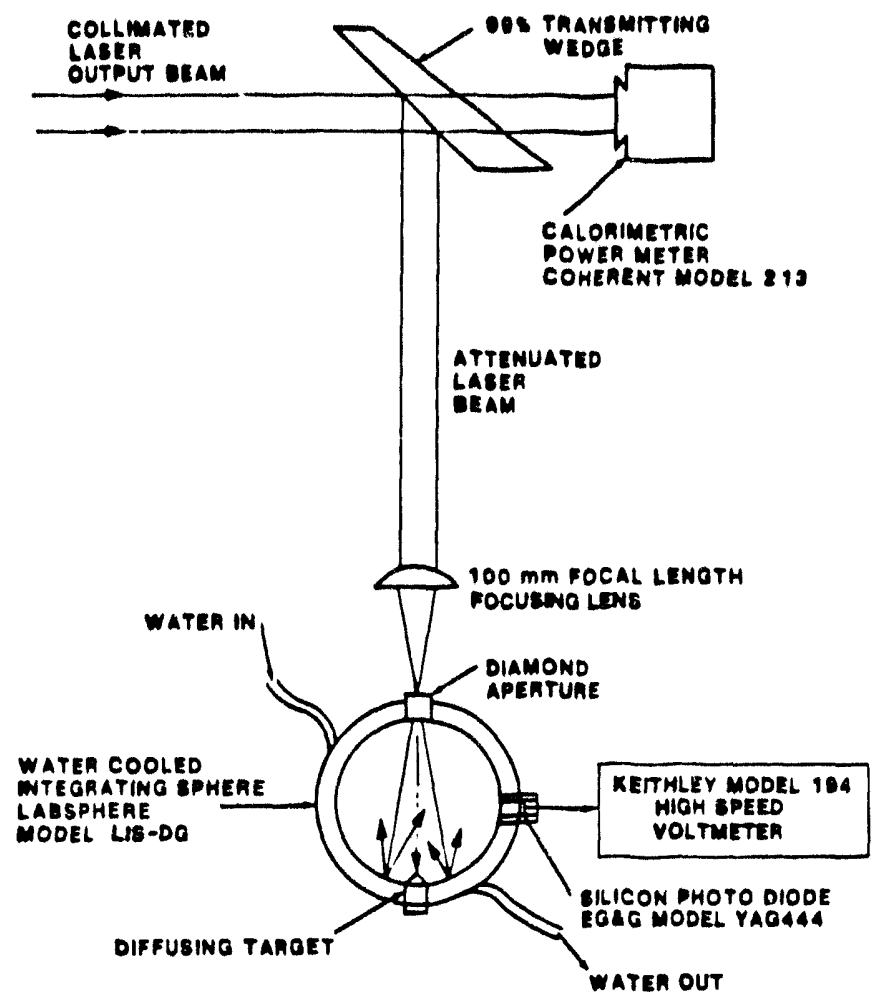

Figure 3-Schematic diagram of diamond aperture spotsize measuring instrument. Set-up for pulsed Nd:YAG laser welding.

The reasons for the difficulty in comparing large and small diameter beam sizes is thought to be due to the necessity of using different size pinholes for the two measurements. There is a specified range of areas that each pinhole is capable of scanning. One of these areas is selected during the set-up before a measurement. The manufacturer states that significant error can result if the fill factor (the ratio of the beam width to the width of the area scanned by the instrument during a measurement) is not kept within a specific range. We found this to be the case, but unfortunately it was not always physically possible to maintain the required fill factor with a large diameter beam. Unfocused beams as well as beams focused with long focal length lenses (questionable results excluded from this report were obtained for $700 \mathrm{~mm}$ and $900 \mathrm{~mm}$ focal length lenses) could not be well characterized by the pinhole size/fill factor limitation.

In addition to the fill factor limitation, the separate steps required to measure beamquality with the above procedures were found to be cumbersome. Multiple repositioning of the detector aperture relative to the lens is required for each of these measurements, and more importantly, several unrecorded spotsize measurements are necessary in order to locate the detector in the proper position to make these required measurements correctly. Since every measurement requires significant sfort, the discarding of useful data seemed illogical. 
The above method was quickly abandoned when we found that measurements close to the focal plane could be used to calculate the propagation constants with the assistance of a personal computer. Software run on an Apple Macintosh II PC was used tn fit a curve to the resulting beam spotsize and focal length position measurements. The curve fitting software used 2 created a nonlinear fit of the data to the exact form of equation [1]. Since all of the data points recorded could be used in this analysis technique, it was decided that the most straightforward experimental method would be to take many spotsize measurements at specific increments above and below the focal plane and not to attempt to locate the exact minimum spotsize position. After initial analysis revealed that replicate measurements did not provide a better fit to the data, measurements with closer $z$ axis position spacings were chosen instead. For the short focal length lenses these increments were either 0.25 or $0.38 \mathrm{~mm}$. All of the focal plane measurements that are reported here were made with a $20 \mu \mathrm{m}$ pinhole with fill factors that ranged from 0.3 to 0.9 .

Since early measurements indicated that $\mathrm{CO}_{2}$ laser power did not affect the focus spot radius to any consistent degree, (i.e., average beam-quality was about the same at all power levels), all reported measurements for the $\mathrm{CO}_{2}$ laser were made at a nominal output power of 300 watts.

To verify the Prometec measurements of the $\mathrm{CO}_{2}$ laser and to make a comparative beam-quality measurement on a Raytheon model SS-500 pulsed Nd:YAG laser, a specially constructed diamond aperture measurement instrument was also used. The instrument is illustrated in Figure 3 . The $1 / \mathrm{e}^{2}$ spotsize was determined by first measuring the transmitted energy using an aperture larger than the spotsize and then substituting smaller apertures until approximately $86 \%$ of the beam was transmitted. The beam is assumed to be circularly symmetrical. The actual $1 / \mathrm{e}^{2}$ spotsize is calculated using equation [3] (Ref. 8) where $T$ is the fractional transmission, $r$ is the radius of the aperture, and $w$ is the $1 / \mathrm{e}^{2}$ spot radius.

$$
T=1-\exp \left[-2(r / w)^{2}\right]
$$

Gold coated diamond wire drawing dies are used for apertures since diamond has a very high thermal diffusivity as well as an extremely low thermal expansion coefficient. The focused laser beam is transmitted through the aperture and collected by an integrating sphere which eliminates any diffraction effects and evenly distributes the laser light around the sphere's diffuse inner surface. For both laser types, the focused beam had to be attenuated to prevent damage to the apertures. A beamsplitter was used to attenuate the $\mathrm{Nd}$ :YAG laser, and a rotating chopper wheel was used to attenuate the $\mathrm{CO}_{2}$ laser, which created a pulsed signal. To measure the laser energy transmitted through the aperture, a detector is attached to one of the exit ports of the sphere. For the Nd:YAG laser measurements, a silicon photodiode was used, and a pyroelectric detector was used for the $\mathrm{CO}_{2}$ laser. The transmitted energy was measured on a high speed voltmeter since both the $\mathrm{Nd}$ :YAG and $\mathrm{CO}_{2}$ signals were pulsed waveforms.

\section{Results and Discussion}

Figure 4 shows the functional relationship between focused spot radius and distance to a nominal 2.5 in focal length aspheric lens. The spot radius was measured with the Laserscope at eight equally spaced but otherwise arbitrary locations on both sides of the focal plane. The non-linear curve fit (eqn. [1] in Fig. 1) to the data in the figure directly gives the laser beam-quality, the minimum spot radius position, and the minimum spot radius. One can see from the figure that the curve fit is effective in averaging the

${ }^{2}$ Kaleidagraph from Synergy Software, Reading, PA. Similar curve fitting programs are available for IBM compatible personal computers. 
variability in the data. It is also apparent that diffraction effects are not an issue with these data, since the variability above and below the focal plane is about the same. It is also clear from Figure 4 that there was little variability in spot radius for the replicated measurements made at each position.

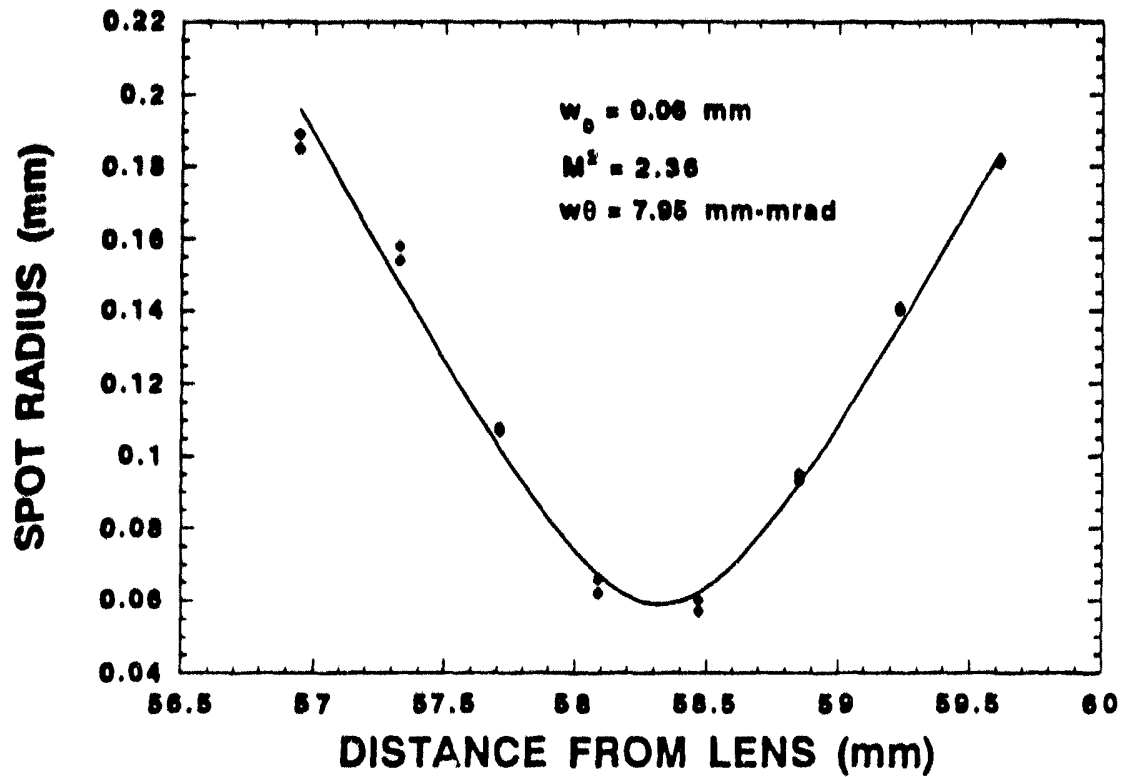

Fig. 4-CO2 laser spotsize for a 2.5 in aspheric lens. Measured with the Laserscope instrument.

The excellent fit of the data that was obtained with the curve fitting software raised the question as to how many datapoints are necessary to characterize a focusing lens and measure the beam-quality. Since each datapoint requires a significant amount of time and effort, a determination of the sample size required is an important practical matter. Figures 5(a) and 5(b) illustrate how few datapoints are actually required to determine the focused spot radius and beam-quality for a 7.5 in focal length plano-convex lens. Both Figure 5(a) and 5(b) are curve fits of the same data but with a large set, [Fig. 5(a)] and a small set [Fig. 5(b)] of datapoints. The two sample sizes yield approximately the same result. Note that to minimize fitting error, at least three datapoints should be taken, with at least one datapoint on each side of the focal plane. Clearly, one of the most important advantages of this curve fitting technique is that neither an exact determination of the minimum spot radius nor it's position is required for the curve fit to be made. While it is certainly preferable to collect data at the focal plane, an exact determination of the focal plane location is often difficult and actually may not be feasible with some instruments.

The diamond aperture instrument was also found to be readily adaptable to the PC based curve fitting analysis. Measurements of focus spot radius for a pulsed Nd:YAG materials processing laser are given in Figure 6. As was the case in Figure 4 for the $\mathrm{CO}_{2}$ laser, several datapoints taken on both sides of the focal plane were used to obtain a nonlinear curve fit and thereby determine the propagation constants for this laser. Note that the $\mathrm{M}^{2}$ value reported for the Nd:YAG laser is much higher than the value given for the $\mathrm{CO}_{2}$ laser. This is of course due to the significant difference in operation characteristics for the two laser types. However, the performance advantage of the $\mathrm{CO}_{2}$ laser is overstated by the use of the $\mathrm{M}^{2}$ parameter. It is important to note that the intrinsic focusability of the two lasers is really determined by the $w_{0} \theta$ product also given in the 

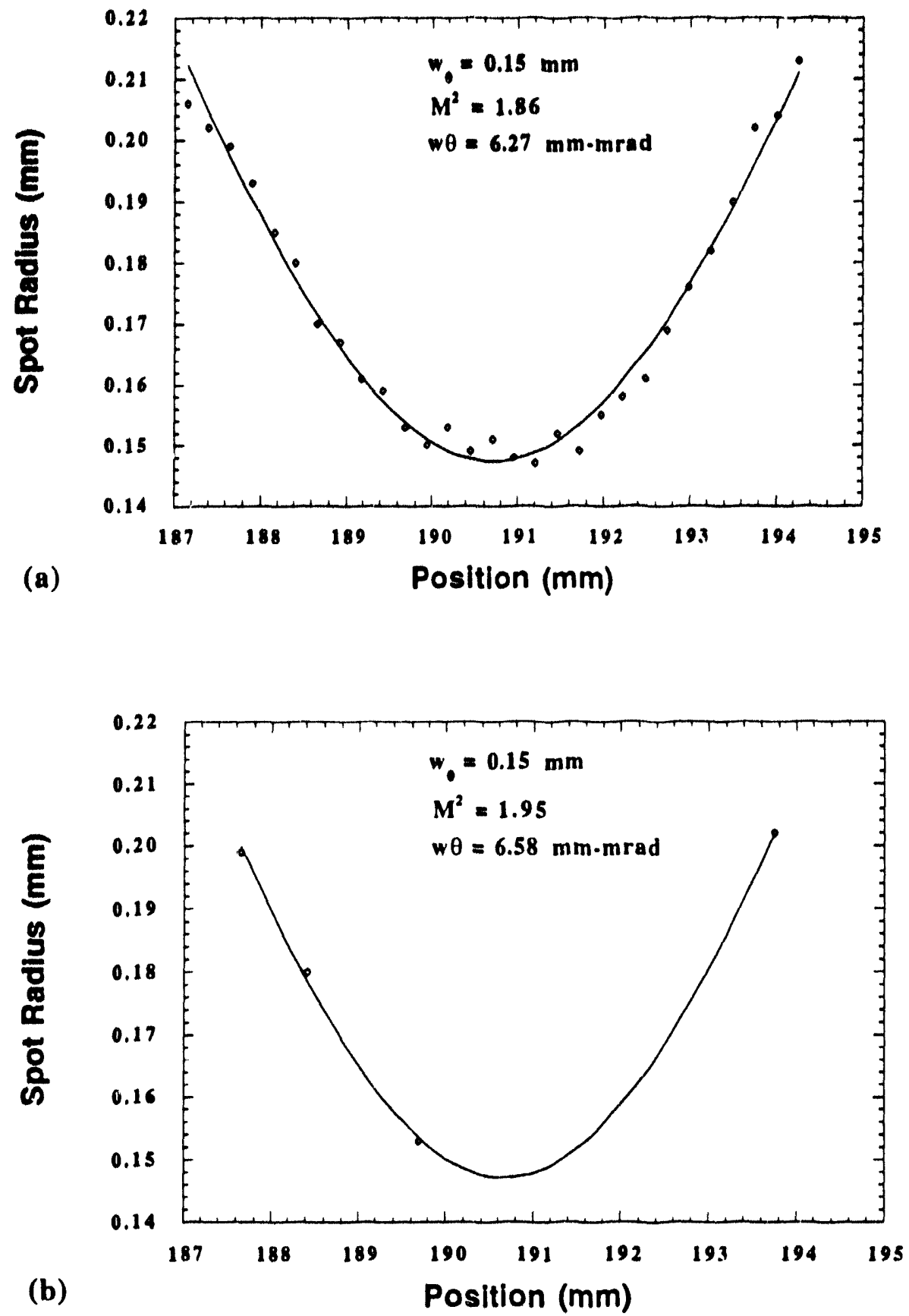

Fig. 5-Curve fitting results for $\mathrm{CO} 2$ laser with a 7.5 in PC lens. (a) large set of datapoints (b) small set of datapoints 
two figures. In other words, to easily and more realistically compare laser beam-quality, the $w_{0} \theta$ product should be given.

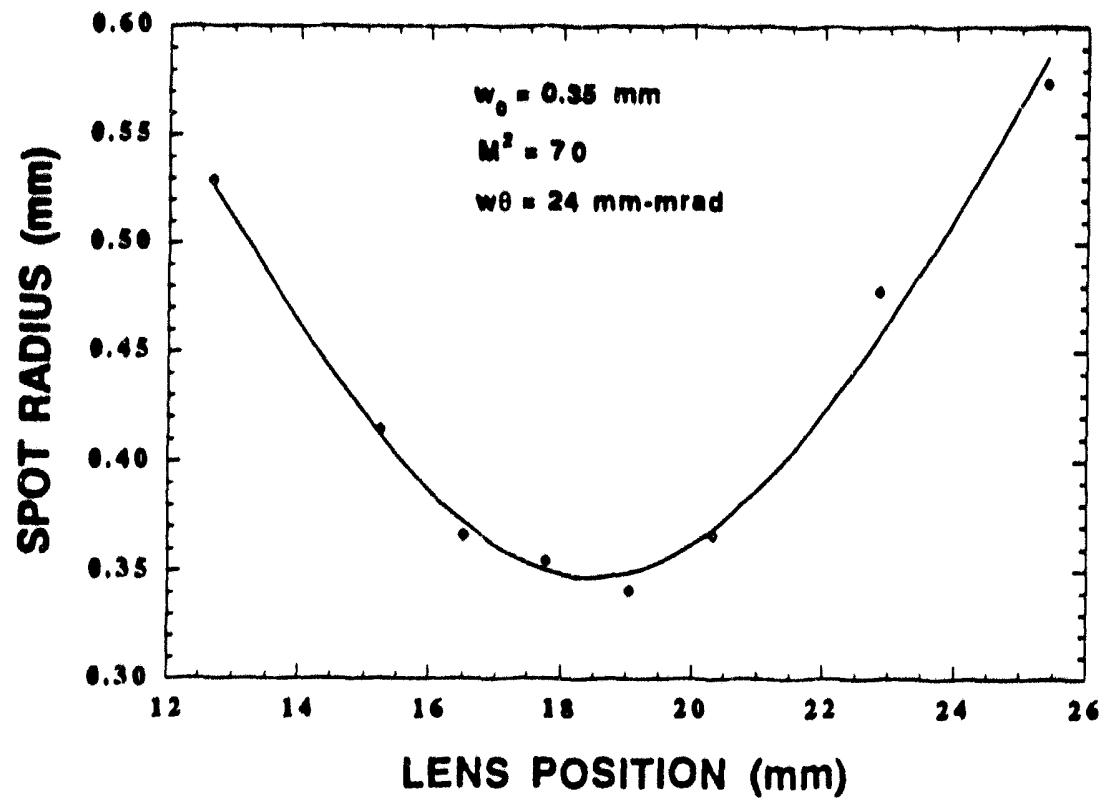

Fig. 6-Pulsed Nd:YAG laser spotsize and beam-quality curve fit, measured with diamond aperture instrument.

The curve fitting analysis is especially well suited to the aperture measurement instrument, because apertures of the exact size required may not be available to directly measure the focal plane region. Using the curve fitting analysis technique, one can easily determine the minimum spot radius and the propagation constants without a direct measurement of the minimum spot radius.

Table I

Resulting Propagation Parameters for ISO and Curve Fit Analyses

\begin{tabular}{|c|cc|cc|cc|}
\hline & \multicolumn{2}{c}{10.0 In Men. Iens } & \multicolumn{2}{c|}{5.0 In Men. Iens } & \multicolumn{2}{c|}{5.0 In PC lens } \\
& 150 & Flt & ISO & Fit & 1SO & Flt \\
\hline$W_{0}(\mathrm{~mm})$ & 0.241 & 0.260 & 0.108 & 0.112 & 0.104 & 0.106 \\
\hline $20(\mathrm{~mm})$ & 257.0 & 256.2 & 124.5 & 124.5 & 126.3 & 126.3 \\
\hline$M^{2}$ & 2.65 & 2.64 & 2.12 & 2.17 & 2.06 & 2.09 \\
\hline
\end{tabular}

A comparison of beam propagation constant values calculated using both the curve fitting and the ISO analysis procedures are given in Table I for three different lenses. The values reported for each lens were calculated using the same set of experimental measurements. The number of measurements used in each calculation of course depends on the technique used, as was described earlier. While only three comparisons of the two analysis techniques are reported here, there is close agreement between the propagation constants determined. Certainly, the use of the same set of measurements for the two analysis techniques has contributed to the good agreement. It appears that the greater difficulty of the ISO method is the restriction of measurement position to a specific region about the focal plane. As discussed earlier, this restriction requires the use of different window sizes with the Laserscope and therefore measurements with increased 
error will follow. As a result measurements with lenses of focal lengths greater than 5.0 in could not be made. However, the diamond aperture measurement instrument does not suffer from the fill factor limitation, and the results for a 10.0 in meniscus lens given in Table I were measured with the diamond aperture instrument.

For the limited results with the aperture measurement instrument reported here, the ISO analysis technique appears to be an acceptable approach for determining propagation parameters. However we prefer the curve fitting analysis technique for beam characterization, because of the experimental difficulties with the ISO approach noted above, and because with the curve fitting analysis the uncertainty in determining the minimum spotsize is not compounded by the uncertainty in determining its position.

The differences between the two measurement instruments do not just pertain to experiment procedural and application difficulties but also to the measurement results. Comparative measurements using the two instruments and made with the same lens and average power are shown in Figure 7 . It is apparent that the two instruments do not yield the same spot radius. The minimum spot radius measured with the diamond apertures is $37 \%$ larger than the minimum spot radius measured with the Laserscope. Similar differences were found for measurements made with a 7.5 in focusing lens at other laser powers. This substantial disparity in srotsize is difficult to explain. The diamond apertures have been inspected for dimensional accuracy and found to have a variation of $\pm 0.008 \mathrm{~mm}$ in aperture diameter, a variation that is too small too account for the discrepancy. No similar calibration data was furnished with the Laserscope by the manufacturer. One must consider that the apertures in both instruments are subject to dimensional variation during the measurement event due to laser beam absorption. Since diamond has a significanlty lower thermal expansion coefficient than the copper used to fabricate the hollow needle, it seems unlikely that the diamond apertures are expanding more than the Laserscope pinhole. Particularly since the measurement exposure times were kept to a minimum with both instruments.

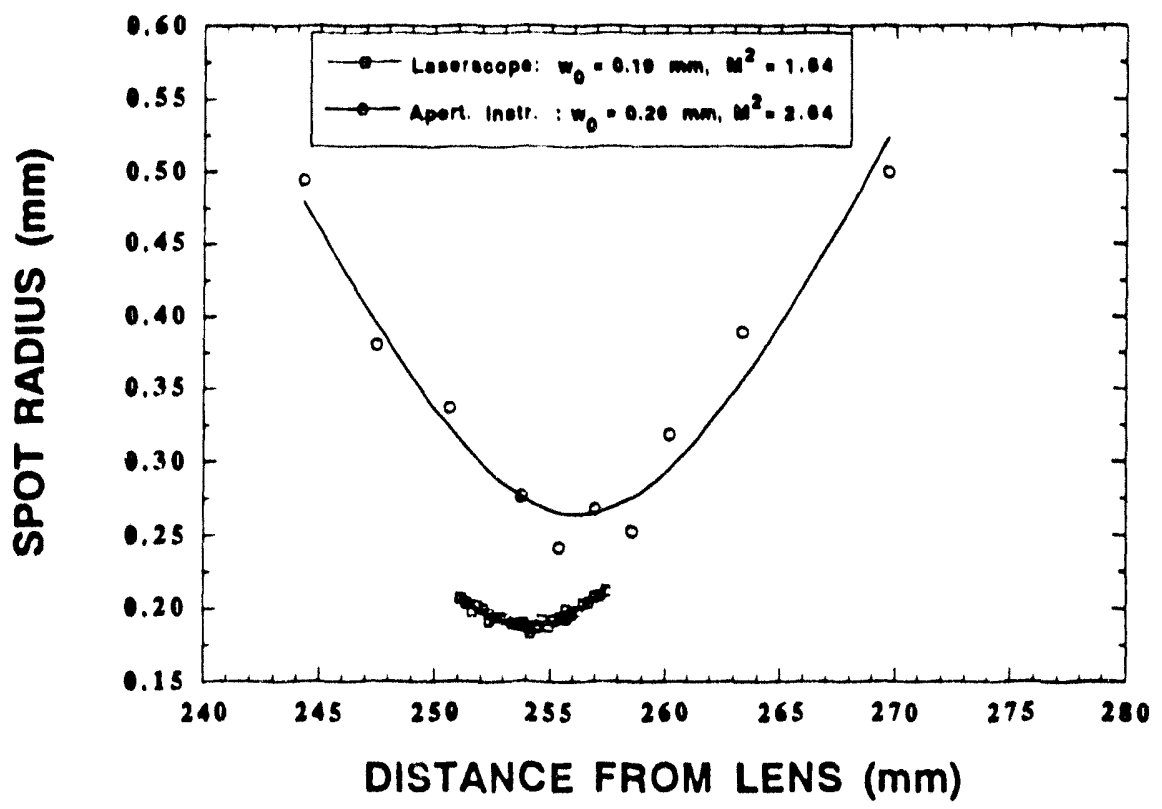

Fig. 7-Comparison of spotsize measurement results for Laserscope and diamond aperture instruments. 10.0 in meniscus lens, 300 watts. 


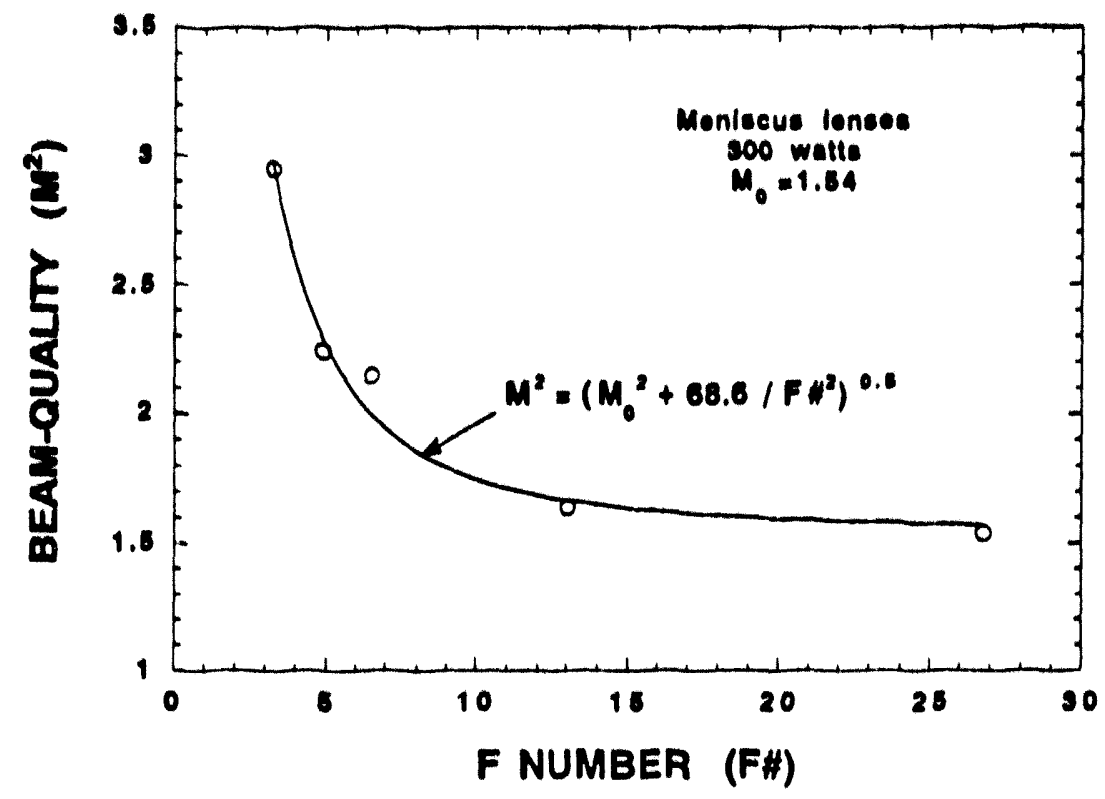

Fig. 8-Dependence of laser beam-quality on the $F$ number of the focusing optic for a $\mathrm{CO}_{2}$ materials processing laser.

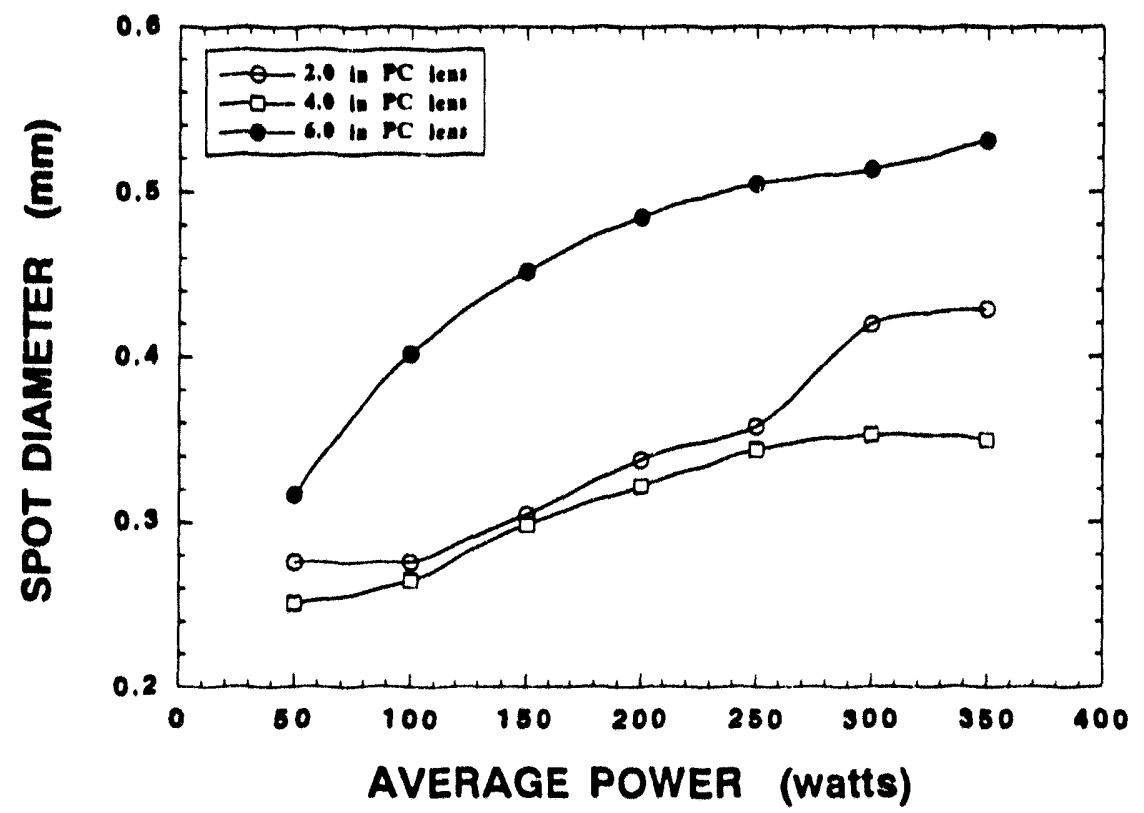

Fig. 9-Degradation in performance of plano-convex lenses due to spherical aberration for a pulsed Nd:YAG laser. 
The importance of lens focal length in the determination of laset heam-quality became paramount during this study. Initial measurements of heam-quality indicated that the laser beam-quality was decreasing with increasing lens focal length. As a result, focusing lenses were obtained with long focal lengths that would not produce sufficient power density for welding but which would likely have minimum spherical aberration. The dependence of measured laset beam-quality on the $F$ number of meniscus focusing lenses is shown in Figure 8. One can see from the figure that heam-quality asymptotically approaches a minimum value at large F numbers. Considering that beam. quality is really an intrinsic property of a laser that should remain constant at a given power, this effect is likely due to spherical aberration of the laser beam hy the focusing optic, since spherical aberration decreases with increasing F number. (Ref. 8) The equation given in the figure is an empirical fit to the data, similar to the form of an equation given by Siegman (Ref, 9). Clearly the F number of the focusing optlc used io, measure the spot radius must be considered in any reported values of beam-quality for a specific laser. The proposed ISO standard addresses this issue and requires focusing optics to have a minimum F number of 16 (Ref. 10).

It is also important to realize, that a knowledge of laser heam-quality does not necessarily permit one to estimate the minimum spot radius using any focal length optic since the effect of spherical aberration is likely to significantly degrade li's performance. This fact is clearly demonstrated in Figure 9 for spot diameter measurements made using three different focal length lenses with the pulsed Nd:YAG laser. These measuremenis were made with the diamond aperture instrument and show that because of spherical aberration effects, the decreased spot diameter one would expect with the shortest focul length lens cannot be achieved with conventional plano-convex oplics. Indeed, for some average power conditions the measured spot diameter with the 2.0 in focal lengih lens is greater than the 4.0 in focal length lens spot diameter.

\section{Conclusions}

1. A personal computer based curve fitting analysis technique was found to be an experimentally simple and analytically powerful method 10 calculate laser beam. quality and the propagation parameters for materials processing type lasers.

2. Laser beam-quality propagation parameters calculated using the proposed isO analysis technique and the curve fitting analysis technique were approximately the same for the lenses compared in this study.

3. The ISO restriction of laser spot radius measurements to specific regions ahout the focal plane limits its utility and application for beam-quality measurements, especially with the Laserscope instrument.

4. The measured spot radius for a given lens was found to he significantly larger with the diamond apertures instrument than with the Laserscope instrument.

5. The F number of the focusing lens was seen to significanily affect both laser beam: quality measurements and the minimum spot diameter one would estimate for a given lens because of the negative effect of spherical aherration.

Acknowledgments

The authors would like to express their sincere appreciation to Fred Hooper for his conscientious assistance in performing these measurements. We would also like to thank Phil Thacher for a careful review of this manuscript.

This work performed at Sandia National Laboratories supported by the U.S. Dept. of Energy under contract number DE-AC04-94AL85000. 


\section{References}

1. Fuerschbach, P. W. 1991; Melting Efficiency in Fusion Welding. In The Metal Science of Joining. TMS: Cincinnati, Ohlo, pp 21-29.

2. Stegman, R. L.; Schriempf, J. T. 1973. Experimental Studies of Laser Supported Absorpilon Waves with $5 \mathrm{~ms}$ Pulses of $10.6 \mu \mathrm{m}$ Radiation. J. Appl. Phys. 44(8)

3. Slegman, A. E. 1990; New Developments in Laser Resonators. In Optical Resonalors. SPIE: LosAngeles, Ca, pp 2.14.

4. Wrighi, D. L. 1992; Status of ISO/TC 172/SC9/WG1 on Standardization of the Mensurement of Beam Widths, Beam Divergence, and Propagation Factor. In Laser Energy Distribution Profiles; Measuremnt and Applications. SPIE: Boston, Massachuselis, pp 2-17.

S. Pope, L. E.; McDonald, T. G. 1981. Nd:YAG Laser Beam Diagnostics, Sandia Nallonal Laboratories, SAND81-0011C.

6. Fuerschbach, P. W. 1987; Piocess Control Improvements in Pulsed Nd:YAG

Laser Closure Welding of Electromechanical Relays. In International Power Beam Conference. ASM: SanDiego, $\mathrm{Ca}$, pp 157-164.

7. Murray, J. E. 1975. High Efficiency TEM00 Mode Nd:YAG Laser Study Program, Ait Force Avionics Laboratory, AFAL-TR-74-339.

8. Hull, D. M.i Stewart, A. In Lasers and Applications. 1985; pp 75-80.

9. Siezman, A. E.; Ruff, J. 1992; Effects of Spherical Aberration on Laser Beam

Quality. In TCALEO. SPIE: pp 130-139.

10. Personal communication with David L. Wright, Spectra Physics Lasers. 



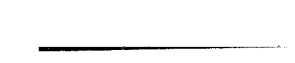

1

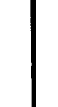

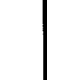

